\title{
Capacidade funcional de idosos com e sem medo de cair
}

Functional Capacity of Elderly

With and Without Fear of Falling
FisiSenectus. Unochapecó Ano 4, n. 2 - Jul/Dez. 2016 p. $22-29$

Paula Cristina Faria Lana. paulalana42@yahoo.com.br Fisioterapeuta. Centro Universitário de Belo Horizonte - UNI-BH.

Sabrina Aparecida Silva. sabrinasyllva1@hotmail.com Fisioterapeuta. Centro Universitário de Belo Horizonte - UNI-BH.

Mariana Lúcia Costa Castro. mariple13@hotmail.com Fisioterapeuta. Centro Universitário de Belo Horizonte - UNI-BH.

\section{Resumo}

Objetivo: Avaliar a capacidade funcional de idosos com e sem medo de cair. Materiais e métodos: Sessenta e um idosos foram divididos em dois grupos segundo os escores obtidos na Falls Efficacy Scale International (FES-I-Brasil) e foram avaliados quando à capacidade funcional ao realizar o teste Short Physical Performance Battery (SPPB), que avalia força, equilíbrio e velocidade da marcha. Para as comparações entre os grupos, foi utilizado o teste $t$ para amostras independentes e o teste de Pearson para investigar possiveis correlações entre os escores da FESI-Brasil e demais variáveis $(p \leq 0,05)$. Resultados: Trinta e sete idosos atingiram nota $\geq$ a 23 na FES-I-Brasil e foram alocados no Grupo 1 (G1, n=37; idade $=70,88 \pm 0,88$ anos), e 24 idosos foram classificados como sem medo de cair e foram alocados no Grupo $2(G 2, n=24$; idade $=69,33 \pm 0,90)$. Os grupos foram diferentes em relação à velocidade da marcha $(G 1: V M=3,10 \pm 0,09 \mathrm{~m} / \mathrm{s}$ e $\mathrm{G} 2: \mathrm{VM}=0,93 \pm 0,03 ; p 0,01)$ e ao número de doenças $(G 1=4,13 \pm 0,21$ doenças e $G 2=3,00 \pm 0,44$ doenças; $p$ 0,01). Houve correlação entre a FESI e número de doenças (FESI x VM p 0,03 r -0,61 e FESI x doenças $p$ 0,01 r 0,58). Conclusão: Este estudo identificou que idosos da comunidade com medo de cair possuem maior número de doenças e andam mais devagar.

\section{Palavras-chave}

Idosos; Medo; Quedas; Autoeficácia; Funcionalidade.

\section{Fisißenectus}




\begin{abstract}
Objective: evaluate the functional capacity of elderly with and without fear of falling. Materials and methods: Sixty-one elderly were divided into two groups according to the score obtained in the Falls Efficacy Scale International - FES-I and were evaluated related to the functional capacity to perform Short Physical Performance Battery (SPPB) which measures the strength, balance and gait speed. For comparisons between groups the t test was used for independent samples and the test Pearson test to investigate possible correlation the scores of FESI and other variables $(p \leq 0,05)$. Results: Thirty-seven elderly achieved score $\geq 23$ on the FES-I and were allocated in Group 1 ( $G 1, n=37$; age $=70,88 \pm 0,88$ years) and 24 elderly were classified as without fear of falling and they were allocated in Group 2 ( $G 2, n=24$; age $=69,33 \pm 0,90$ years). The groups were different in relation to gait speed ( $G 1, G P: 3,10 \pm 0,09 p$ 0,02; GP(m/s): $93 \pm 0,03 p$ 0,01; diseases: $4,13 \pm 0,21 p 0,01)$ and the number of diseases. There was correlation between the FES-I and the number of diseases (FESI x GP p 0,03 r 0,61 e FESI x disease $p$ 0,01 r 0,58). Conclusion: This study found that of older adults with fear of falling have more diseases and move more slowly.
\end{abstract}

\title{
Keywords
}

Elderly; Fear; Falling; Self-efficacy; Functionality.

\section{Introdução}

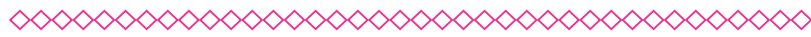

0 aumento populacional de idosos é um fenômeno universal que ocasiona mudanças na pirâmide etária, sendo o Brasil o sétimo lugar na lista dos países mais envelhecidos do mundo. Estima-se que em 2025 o nosso país ocupe a sexta posição $0^{1,2}$. A capacidade funcional é considerada como um importante marcador do envelhecimento bem-sucedido e da qualidade de vida dos idosos. Pode ser definida como habilidade de manter as atividades mentais e físicas necessárias, ou seja, o idoso pode viver sem ajuda para as atividades básicas de vida diária (AVD) e as atividades instrumentais $(A I V D)^{3,4}$

O comprometimento da capacidade funcional pode estar associado à predição de fragilidade, dependência, institucionalização, maior risco de quedas, morbidade e mortalidade, acarretando, assim, em complicações na terceira idade ${ }^{4}$. A capacidade funcional pode ser avaliada por meio do teste Short Physical Performance Battery (SPPB), capaz de avaliar o equilíbrio, a velocidade da marcha e a força muscular. 0 teste possui o escore total obtido pela soma dos três testes, visto que quanto maior a nota, melhor o desempenho ${ }^{5}$.

0 medo de cair é descrito como um sentimento de grande inquietação ante a noção de um perigo real, aparente ou imaginário de quedas ${ }^{1}$. Atualmente, os estudos têm definido o medo de cair como o baixo senso de autoeficácia ${ }^{5,6}$. A autoeficácia é entendida como autoconfiança. Pessoas com grande senso de autoeficácia normalmente são capazes de superar situações desafiadoras, focando-se mais nas atividades do que nos obstáculos. Já as pessoas com baixo senso de autoeficácia tendem a focar-se mais nas suas limitações, enfatizando suas dificuldades ${ }^{6}$.

O medo de cair tem sido incluído em um círculo vicioso, que inclui o risco de quedas, o déficit de equilíbrio e mobilidade, o declínio funcional e da qualidade de vida, repercutindo em mais medo $0^{6}$. 0 senso de autoeficácia relacionado às quedas pode ser avaliado pela Falls Efficacy Scale-International (FES-I Brasil), que verifica a preocupação com a possibilidade de cair ao realizar 16 atividades de vida diária. 0 escore total é dado pela soma de todas as atividades perguntadas, e uma pontuação igual ou maior a 23 identifica o idoso com risco potencial de quedas ${ }^{1}$.

Atualmente, o medo de quedas vem sendo reconhecido como um problema de saúde para a população idosa, inclusive naqueles idosos que nunca caíram, o que pode levar à redução das atividades funcionais, associado a desfechos variados, como quedas, declínio funcional e depressão. A redução das atividades funcionais por medo de cair, em níveis excessivos, pode reduzir a interação social, gerar inatividade física e comprometer a qualidade de vida9. Logo, o objetivo do presente estudo foi de comparar a capacidade funcional de idosos com e sem medo de cair. 


\section{Materiais e métodos}

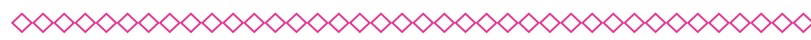

Tratou-se de um estudo observacional com delineamento transversal, aprovado pelo Comitê de Ética em Pesquisa do Centro Universitário de Belo Horizonte (UNI-BH), MG, Brasil (Parecer 44967815.0.0000.5093). Os voluntários foram idosos da comunidade de Belo Horizonte e região metropolitana. Para o recrutamento dos voluntários, os membros da equipe do projeto fizeram uma divulgação informando que estava sendo feito um trabalho com os idosos da comunidade, alertando o dia, horário e local da coleta. Assim, os pesquisadores esperavam os voluntários e os abordavam convidando-os a participar do estudo. As avaliações dos voluntários foram realizadas na Clínica Integrada da Saúde do Centro Universitário de Belo Horizonte (UNIBH) e em espaços apropriados para coleta que fossem capazes de reproduzir, da melhor forma possível, o espaço da clínica do UNIBH. Foi tomado o cuidado para que o ambiente fosse adequado para a execução dos testes; como, por exemplo: o piso deveria ser plano e regular e poder ser demarcado, os assentos deveriam estar em bom estado, era necessária boa iluminação, e o ambiente sem riscos de acidentes, entre outros detalhes. 0 período da coleta de dados foi de outubro de 2015 a fevereiro de 2016.

Foram adotados os seguintes critérios de inclusão: possuir idade igual ou superior a 60 anos; ambos os sexos; ser capaz de levantar-se da cadeira, ficar de pé e andar a distância de 8 metros sem ajuda e apresentar estado cognitivo preservado segundo o Mini-Exame do Estado Mental (MEEM 13, para analfabetos; 18 , para indivíduos com um a sete anos de escolaridade; e 26, para oito anos ou mais de escolaridade) ${ }^{8}$. Ainda, foram excluídos do estudo aqueles que estavam em tratamento fisioterápico no período da coleta de dados; que tivessem doenças neurológicas, cardiovasculares e/ou musculoesqueléticas que impossibilitassem a realização dos testes.

Por cuidados metodológicos, os examinadores realizaram um treinamento antes do início da coleta de dados, no qual foi estipulada a melhor forma de realizar as perguntas do questionário, da entrevista da FESI-Brasil e a execução do SBBP, de forma que todos os examinadores adotassem os mesmos critérios entre si. Sendo assim, estabeleceu-se um passo a passo que foi seguido com todos os voluntários.

Os voluntários foram submetidos a uma única entrevista. Inicialmente, responderam a um questionário clínico e demográfico elaborado pelos pesquisadores do estudo, contendo perguntas como: dados pessoais, doenças associadas, medicamentos em uso, medidas antropométricas, histórico de quedas e entre outros. Em seguida, foi aplicada a escala FES-I-Brasil ${ }^{1}$ sob a forma de entrevista, para avaliar o medo de cair; e, finalmente, os participantes executaram o teste SPPB ${ }^{5}$ para avaliar a capacidade funcional. Após a coleta dos dados, a amostra foi divida em dois grupos. Idosos com o escore igual ou superior a 23 na FES-I-Brasil ${ }^{1}$ foram incluídos no grupo com medo de cair (G1); e aqueles que obtiveram escore menor que 23 foram incluídos no grupo sem medo de cair (G2).

\section{Instrumento para coleta Short Physical Pergormance Battery}

A capacidade funcional foi avaliada pelo teste Short Physical Performance Battery (SPPB) versão brasileira, composto por três testes que avaliam o equilíbrio, a velocidade de marcha e a força muscular ${ }^{5}$. 0 teste será descrito a seguir:

- Primeiramente, avalia-se o equilíbrio estático do indivíduo em três posturas distintas: de pé com os pés unidos, na postura semitandem e na postura tandem, devendo o voluntário permanecer por 10 segundos nas posturas. É importante ressaltar que o voluntário somente irá realizar as três etapas do teste, caso passe na primeira e assim sucessivamente.

- Na segunda etapa do teste é determinada a velocidade da marcha, quando o voluntário caminhará com passos habituais em um percurso plano e regular de 8 metros e o tempo gasto para a execução do teste será cronometrado no percurso de 4 metros, desconsiderando-se os 2 metros iniciais e finais, por se tratarem das fases de aceleração e desaceleração da marcha. A velocidade da marcha será, então, determinada dividindo-se a distância percorrida pelo tempo gasto para percorrer os 4 metros. 
- A terceira e última etapa consiste em avaliar a força muscular de membros inferiores, realizado por meio do teste Senta e Levanta, quando o voluntário irá sentar e levantar de uma cadeira por cinco vezes consecutivas, sem o auxilio dos membros superiores, com registro do tempo gasto para sua realização.

Para cada etapa do teste, o voluntário recebe uma pontuação de acordo com a execução da tare$\mathrm{fa}$, do tempo e da capacidade de realizar. 0 escore total do SPPB é obtido através da somatória entre os resultados dos testes aplicados, sendo a pontuação classificação de zero (pior desempenho) a 12 (melhor desempenho) ${ }^{5}$.

\section{Falls Efficacy Scale-International}

A autoeficácia relacionada às quedas foi medida pela versão brasileira da Falls Efficacy Scale-International (FES-I-Brasil), que avalia a preocupação com a possibilidade de cair ao realizar 16 atividades, com escores de 1 a 4 pontos por atividade (sendo 1: nem um pouco preocupado; 2 : pouco preocupado; 3: muito preocupado; e 4: extremamente preocupado) ${ }^{1}$. 0 escore total é obtido pela soma da pontuação em todas as atividades. Atualmente, uma pontuação maior ou igual a 23 pontos indica o idoso com risco potencial de queda ${ }^{1}$.

\section{Análise estatística}

Foi feito cálculo amostral utilizando dados de estudos anteriores que tiveram como desfecho principal os resultados da escala de autoeficácia FESI-Brasil, determinando a necessidade de 50 voluntários. Foram utilizadas análises descritivas da amostra, com valores de média e desvio-padrão para as variáveis numéricas. A normalidade dos dados foi avaliada pelo teste de Kolmogorv-Smirnov, e todas as variáveis foram consideradas com distribuição normal, justificando a utilização da estatística paramétrica. Para as comparações entre os grupos com e sem medo de cair, utilizou-se o teste $t$ para amostras independentes. 0 teste de correlação de Pearson foi utilizado para identificar possiveis correlações entre os escores da FES-I-Brasil e números de doenças e os escores do SPPB. Todas as análises foram realizadas no programa estatístico SPSS 17.0 e foi considerado o nível de significância de 0,05.

\section{Resultados}

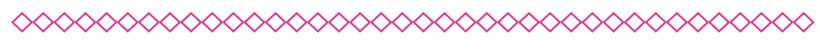

Neste estudo, dos 61 idosos avaliados, 73,7\% $(n=45)$ eram do gênero feminino e $26,2 \%(n=16)$ do gênero masculino. Os voluntários foram divididos em dois grupos. O G1 (com medo de cair) composto por 37 idosos com idade média de 70,88 $\pm 0,88$; e o $\mathrm{G} 2$ (sem medo de cair) por 24 idosos com idade média de $69,33 \pm 0,90$. Do total da amostra $59 \%(n=36)$ dos voluntários praticavam exercício físico regular e $40,9 \%(n=25)$ afırmaram não praticar exercício físico regularmente. As demais características da amostra estão demonstradas na Tabela 1.

Trinta e sete idosos $(60,6 \%)$ obtiveram nota igual ou superir a 23 na FES-I e foram alocados no grupo 1 . Vinte e quatro idosos $(39,3 \%)$ apresentaram nota abaixo do ponto de corte, sendo alocados no grupo 2. Os resultados da avaliação do desempenho funcional pelo SPPB (escore total e resultado dos testes individuais de velocidade da marcha (VM), equilíbrio e senta-levanta (TSL); e dos valores absolutos da VM e TSL) encontram-se expressos na Tabela 2. Houve diferença significativa no teste de velocidade da marcha do G1 (G1: escore: $3,10 \pm 0,09-G 2: 3,96 \pm 0,08 \boldsymbol{p}=0,02)$, e do valor absoluto (m/s) da VM (G1: escore: 0,93 $\pm 0,03-\mathrm{G} 2$ : $1,11 \pm 0,08 \boldsymbol{p}=0,01)$.

Constatamos, no estudo, uma correlação entre a autoeficácia relacionada às quedas medida pela FES-I com a velocidade da marcha (FESI versus VM: $p=0,03$ e $r=-0,61$ ) e no número de doenças (FESI versus $N^{0}$ doenças: $p=0,01$ e $r 0,58$ ) dos voluntários com medo de cair. Ou seja, idosos com medo de cair possuem maior número de doença e andam de forma mais lenta ou vice- versa (Tabela 3 ).

\section{Discussão}

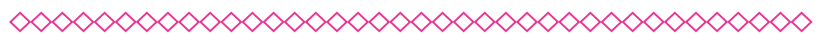

0 presente estudo, realizado com os idosos da comunidade, demonstrou associação entre a velocidade da marcha, número de doenças e o senso de 
autoeficácia para prevenir quedas. Os grupos foram diferentes em relação ao número de doenças e à velocidade da marcha (tanto no escore total do SPPB quanto nos valores absolutos da VM).

Segundo Camargos et al. ${ }^{1}$, a FES-I-Brasil foi adequadamente adaptada para a população de idosos brasileiros da comunidade. É considerado como um instrumento promissor para a avaliação do medo de cair tanto em pesquisas como na prática clínica, por ser de fácil aplicabilidade e durar em torno de 5 a 10 minutos para sua aplicação. De acordo com os resultados obtidos neste estudo, observou-se correlação moderada e negativa $(r-0,61 ; p=0,03)$ entre o senso de autoeficácia para prevenir quedas e a velocidade da marcha e uma correlação moderada e positiva ( $r$ 0,58; $p=0,01$ ) entre o senso de autoeficácia e número de doenças. Ou seja, idosos com medo de cair possuem maior número de doença e andam de forma mais lenta ou vice-versa.

Como descrito anteriormente, houve uma correlação moderada e positiva entre a FES-I-Brasil e o número de doenças dos idosos com medo de cair. Os estudos vêm mostrando que um maior número de doenças, sejam elas agudas ou crônicas, em idosos é um fator importante para a qualidade de vida desta população ${ }^{6,8}$. Sabe-se que idosos com maior número de comorbidades têm mais chances de serem frágeis e apresentarem mobilidade reduzida. 0 medo de cair surge como um dos mecanismos que levam à limitação funcional.

Sabe-se que o medo que cair compromete a capacidade funcional de idosos da comunidade ${ }^{6,9}$; entretanto, quando avaliado nesse estudo (por meio do teste SPPB), não foi encontrada uma diferença significativa entre grupos nos escores do equilíbrio e da força muscular. Pressupõe-se que os resultados possam ter sido influenciados pelas características dos voluntários como idade, prática de atividade física regular e talvez pelo fato de o teste SPPB não ser sensível o suficiente para detectar diferenças na mobilidade dos participantes com e sem medo de cair.

Caminhar é considerada uma atividade funcional complexa, dependente de diversas capacidades físicas, como o estado de saúde do indivíduo, condições musculoesqueléticas, função sensorial e perceptiva, nível de atividade habitual, assim como as suas características ambientais ${ }^{11,12}$. Rezende et al. ${ }^{13}$ definem a marcha como um resultante da interação dos sistemas neurológico, musculoesquelético, vestibular e somatossensorial. A VM é considerada, nos dias atuais, como um importante indicador do estado geral de saúde e vitalidade de pessoas idosas ${ }^{12,15}$.

De acordo com a avaliação funcional, os participantes do G1 apresentaram escore da VM no teste SPPB de 3,10 $\pm 0,09$ e VM média de 0,93 $\pm 0,03 \mathrm{~m} / \mathrm{s}$. Tal resultado nos faz concluir que os idosos que têm medo de cair andam mais devagar. Estudos apontam que idosos que caem durante a marcha podem ter maior risco de incapacidades em longo prazo, devido às consequências físicas, psicológicas e sociais que a queda traz ao idoso, 0 que pode levar à restrição de atividades e à perda da independência ${ }^{14}$.

Apesar de os grupos não apresentarem diferenças em relação ao escore total do SPPB, podemos perceber que idosos com medo de cair andam com VM inferior a $1,0 \mathrm{~m} / \mathrm{s}$ e aqueles sem medo de cair, com VM superior a este valor. Fritz \& Lusar$\mathrm{di}^{16}$ sugerem que idosos com VM inferior a $1,0 \mathrm{~m} / \mathrm{s}$ estão mais propensos a serem dependentes nas AVD's, a serem hospitalizados e a necessitarem de reabilitação para reduzir o risco de quedas, além de serem mais dependentes na marcha fora de casa, o que corrobora os dados alcançados.

Por tamanha relevância nos dias atuais, Studenki et al. ${ }^{15}$ afirmam que a VM pode ser considerada o sexto sinal vital, porque reflete problemas patológicos ocultos e prevê o estado de saúde futuro e possíveis declínios funcionais. Consideram ainda como a "medida quase perfeita", por ser uma ferramenta de rastreio de fácil acesso na prática clínica que deveria ser usada para oferecer informações sobre a capacidade funcional da população idosa ${ }^{17}$. Considerada tão importante quanto a monitorizarão rotineira da pressão arterial, frequência cardíaca, temperatura, frequência respiratória e relato de dor, a VM pode fornecer uma perspectiva funcional relevante para o estado de saúde do idoso ${ }^{13,15}$. Dessa forma, podemos observar uma relação direta com os participantes do $\mathrm{G} 1$ que andaram mais devagar na execução do teste SPPB. 
Como demonstrado nos resultados, observamos, neste estudo, uma correlação moderada e negativa entre os escores da FES-I-Brasil e a velocidade da marcha, indicando que os idosos com pior senso de autoeficácia para prevenir quedas apresentaram marcha mais lenta. 0 desenho do presente estudo não nos permite falar de causa e efeito, mas sabe-se que essa relação é bidirecional, onde idosos com medo de cair podem andar de forma mais lenta e/ou idosos que andam com menor velocidade da marcha podem apresentar medo de cair.

Ressalta-se que o estudo apresenta algumas limitações. A primeira refere-se ao fato de se tratar de um estudo transversal, que impossibilita estabelecer uma relação de causalidade, ou seja, é impossível saber se o medo de cair diminui a velocidade da marcha ou vice-versa, podendo-se apenas destacar a relação entre as variáveis. Além disso, a amostra deste estudo é de conveniência - ou seja, o processo de seleção não foi aleatorizado - e talvez tenha sido composta por idosos mais ativos e independentes, fato que pode ter reduzido a validade interna do estudo. Mesmo assim, os resultados que foram obtidos são de grande relevância para a área da gerontologia, uma vez que estudos que investigam a relação do medo de cair com a força muscular, equilíbrio e marcha ainda são escassos. Outrossim, o conhecimento da relação entre o medo de cair e a velocidade da marcha, podem fornecer subsídios para a melhor avaliação e tratamento da população idosa. Novos estudos como este devem ser realizados com o intuito de fornecer evidências mais robustas acerca dessas relações.

\section{Conclusão}

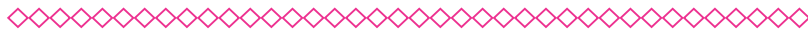

Idosos da comunidade com medo de cair possuem maior número de doenças e andam mais devagar e vice-versa. Além disso, este estudo identificou uma relação entre o medo de cair, a velocidade da marcha e o número de comorbidades. Portanto, a avaliação da velocidade da marcha e do medo de cair devem fazer parte do processo de avaliação e tratamento da população idosa.

\section{Referências}

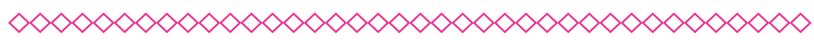

1. Camargos FFO, Dias RC, Dias JMD, Freire MTF. Adaptação transcultural e avaliação das propriedades psicométricas da Falls Efficacy Scale - International em idosos Brasileiros (FESI-BRASIL). Rev. bras. fisioter. [online]. 2010; 14(3):237-43.

2. Lustosa PL, Oliveira AL, Santos SL, Guedes CR, Parentoni NA, Pereira MSL. Efeito de um programa de treinamento funcional no equilíbrio postural de idosas da comunidade. Fisioter Pesq. 2010; 17(2):153-56.

3. Fhon SRJ, Wehbe FCCS, Vendruscolo PRT, Marques SS, Rodrigues PAR. Quedas em idosos e sua relação com a capacidade funcional. Rev. Latino-Am. Enfermagem. 2012 Set/Out; 20(5): [8 telas].

4. Guimarães $T C H L$, Galdino $A C D$, Martins MLF, Abreu RS, Lima M, Vitorino MFD. Avaliação da capacidade funcional de idosos em tratamento fisioterapêutico. Revista Neurociências. 2004 Jul/ Set; $12(3): 130-33$.

5. Marchon MR, Cordeiro CR, Nakano MM. Capacidade Funcional: estudo prospectivo em idosos residentes em uma instituição de longa permanência. Rev. Bras. Geriatr. Gerontol. 2010; 13(2):203-14.

6. Teixeira DC, Oliveira IL, Dias RC. Perfil Demográfico, Clínico e Funcional de Idosos Institucionalizados como História de Quedas. Rev. Fisioterapia em movimento. 2016; 19(2):101-08.

7. Yoshitome AY, Ferreira DCO. Prevalência e características das quedas de idosos institucionalizados. São Paulo: Universidade Federal de São Paulo; 2010.

8. Fernandes MG, Oliveira LRMF, Talitha $K$, Barbosa F, Rodrigues DMM, Bastos AAR. Avaliação do medo de cair em idosos em atendimento ambulatorial. Rev Enferm UFPE. 2013 Abr; 7(4):1160-66.

9. Perracini MR, Fló CM. Fisioterapia: Teoria e prática clínica, funcionalidade e envelhecimento. Rio de Janeiro: Guanabara Koogan; 2009. 
10. Guedes RC, Dias RC, Pereira LSM, Silva SLA, Lustosa LP, Dias JMD. Influence of dual task and frailty on gait parameters of older communitydwelling individuals. Braz J Phys Ther. 2014 Sep/ Oct; 18(5):445-52.

11. Wamser LE, Valderramas RS, Paula AJ, Schieferdecker MEM, Amarante PT, Pinotti F et al. Melhor Desempenho No Teste Timed Up Go Está Associado a Melhor Desempenho Funcional em Idosas da Comunidade. Rev. bras. geriatr. gerontol. [online]. 2015; 9(4):138-43.

12. Van Kan GA, Rolland Y, Andrieu S, Bauer J, Beauchet O,Bonnefoy M, et al. Gait speed at usual pace as a predictor of adverse outcomes in community-dwelling older people: an International Academy on Nutrition and Aging (IANA) task force. J Nutr Health Aging. 2009; 13(10):881-89.

13. Rezende BAA, Silva LI, Cardoso BF, Beresford $\mathrm{H}$. Medo dos idosos em sofrer quedas recorrentes: a marcha como fator determinante da independência funcional. Rev. Acta Fisiartr. 2010; 17(3):117-21.

14. Physical performance measures in the clinical setting. J Am Geriatr Soc. 2003; 51(3):314-22.

15. Studenski S, Perera S, Wallace D, Chandler JM, Duncan PW, Rooney E, et al. Physical performance measures in the clinical setting. J Am Geriatr Soc 2003; 51(3):314-22.

16. Fritz S, Lusardi M. White paper: walking speed: the sixth vital sign. J Geriatr Phys Ther. 2009; 32(2):2-5.

17. Montero-Odasso M, Schapira M, Soriano ER, Varela M, Kaplan R, Camera LA, et al. Gait velocity as a single predictor of adverse events in healthy seniors aged 75 years and older. J Gerontol A Biol Sci Med Sci. 2005; 60(10):1304-09. 


\section{Anexos}

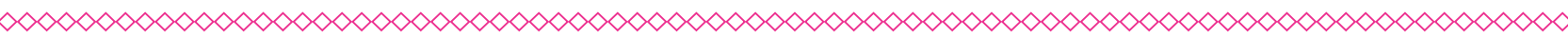

Tabela 1 - Características demográficas, antropométricas e clínicas da amostra.

\begin{tabular}{cccccc} 
Variável & Medo & N & Média & Desvio-padrão & Valor $p$ \\
Idade & G1 & 37 & 70,88 & 0,88 & 0,30 \\
No Doenças & G2 & 24 & 69,33 & 0,90 & $0,01^{*}$ \\
Medicamentos & G1 & 37 & 4,13 & 0,21 & 0,27 \\
IMC kg/m & G2 & 24 & 3,00 & 0,44 & 0,78 \\
& G1 & 37 & 3,79 & 0,43 & 0,50 \\
\hline
\end{tabular}

n: número de Idosos; G1: grupo com medo de cair; G2: grupo sem medo de cair; IMC: Índice de massa corpórea; * nivel de significância positivo do teste t para amostras independentes.

(clique para voltar ao texto)

Tabela 2 - Média e desvio-padrão ( \pm DP) das variáveis do SPPB e do valor absoluto da VM e TSL.

\begin{tabular}{|c|c|c|c|c|c|}
\hline Variável & Medo & $\mathbf{N}$ & Média & Desvio-padrão & Valor $p$ \\
\hline \multirow[t]{2}{*}{ SPPB Total } & G1 & 37 & 8,74 & 0,30 & 0,15 \\
\hline & $\mathrm{G} 2$ & 24 & 9,61 & 0,56 & \\
\hline \multirow[t]{2}{*}{ SPPB EQ } & G1 & 37 & 3,00 & 0,17 & 0,50 \\
\hline & $\mathrm{G} 2$ & 24 & 3,22 & 0,29 & \\
\hline \multirow[t]{2}{*}{ SPPB TSL } & G1 & 37 & 2,18 & 0,18 & 0,10 \\
\hline & $\mathrm{G} 2$ & 24 & 2,72 & 0,28 & \\
\hline \multirow[t]{2}{*}{ SPPB VM } & G1 & 37 & 3,10 & 0,09 & $0,02^{*}$ \\
\hline & $\mathrm{G} 2$ & 24 & 3,96 & 0,19 & \\
\hline \multirow{2}{*}{$\mathrm{VM}(\mathrm{m} / \mathrm{s})$} & G1 & 37 & 0,93 & 0,03 & $0,01^{*}$ \\
\hline & $\mathrm{G} 2$ & 24 & 1,11 & 0,08 & \\
\hline \multirow[t]{2}{*}{ TSL (s) } & G1 & 37 & 15,69 & 0,82 & 0,09 \\
\hline & $\mathrm{G} 2$ & 24 & 13,26 & 0,97 & \\
\hline
\end{tabular}

SPPB: Short Physical Pergormance Battery; EQ: equilíbrio; VM: velocidade da marcha; TSL: teste senta e levanta; VM (m/s): valores absolutos; TSL(s): valores absolutos; *nível de significância positivo do teste $t$ para amostras independentes.

(clique para voltar ao texto)

Tabela 3 - Correlação entre a FESI-Brasil e a velocidade da marcha e número de doenças.

\begin{tabular}{ccc} 
Variável & $p$ & $r$ \\
FESI X VM & $0,03^{*}$ & $-0,61$ \\
FESI X N ${ }^{\circ}$ DOENÇAS & $0,01^{*}$ & 0,58 \\
\hline
\end{tabular}

FESI: Falls Efficacy Scale-International; VM: velocidade da marcha; $p^{*}$ nível de significância; r: índice de correlação entre as variáveis. 